Voix et Images

voixetimages

\title{
Gilles Marcotte, critique et romancier. Entretien
}

\section{André Brochu}

Volume 6, numéro 1, automne 1980

Gilles Marcotte

URI : https://id.erudit.org/iderudit/200247ar

DOI : https://doi.org/10.7202/200247ar

Aller au sommaire du numéro

Éditeur(s)

Les Presses de l'Université du Québec

ISSN

0318-9201 (imprimé)

1705-933X (numérique)

Découvrir la revue

Citer ce document

Brochu, A. (1980). Gilles Marcotte, critique et romancier. Entretien. Voix et Images, 6(1), 5-34. https://doi.org/10.7202/200247ar d'utilisation que vous pouvez consulter en ligne.

https://apropos.erudit.org/fr/usagers/politique-dutilisation/ 


\title{
Gilles Marcotte, critique et romancier
}

\author{
Entretien/André Brochu
}

\section{A - Les commencements}

A.B. D'abord, une question générale: comment en es-tu venu à la littérature? As-tu voulu d'abord être critique, puis romancier? Quand et comment le goût d'écrire s'est-il d'abord manifesté chez toi et comment s'est-il développé?

G.M. A vrai dire dans ma jeunesse, je ne pensais devenir ni critique ni romancier, et je doutais très fort même de pouvoir jamais devenir écrivain. Je me souviens que, lorsque j'étais en philosophie je pense, il y avait une sorte de cercle d'études qui était dirigé par un homme fort intelligent, monseigneur Maltais, qui est devenu recteur de I'Université de Sherbrooke; et je lui avais demandé - c'était le genre de question qu'on pouvait se poser alors : "Est-ce qu'on peut ètre un intellectuel sans écrire? II m'a dit que c'était un petit peu difficile! - parce que, évidemment un intellectuel, en principe, cela doit communiquer. Je raconte cette anecdote uniquement pour te dire que, dans ma jeunesse, l'écriture me paraissait quelque chose d'à peu près impossible. Je n'ai jamais pensé écrire des livres avant ma trentième année. D'abord j'ai fait de la critique, mais je ne suis pas entré dans le journalisme comme critique; $j$ 'ai fait de tout dans le journalisme, parce qu'il fallait que je gagne ma vie. C'est un peu par hasard que je suis arrivé à la critique, mais il est évident que cela m'intéressait. Ensuite, après la trentième année, j'ai publié à peu près en même temps un livre de critique et un roman et les deux se sont faits de façon également fort hasardeuse: le livre de critique, c'est un éditeur, Claude Hurtubise, qui me l'avait demandé et qui m'a presque forcé à le faire; le roman, je l'avais commencé quelques années auparavant, pas dans le but très spécifique d'écrire un roman. II s'agissait avant tout d'éloigner de moi une certaine expérience que j'avais vécue - j'ai passé deux ans au grand séminaire - et non seulement de l'éloigner mais aussi de l'analyser de façon que je puisse arriver à une certaine libération. Donc le grand rêve d'être écrivain, je ne l'ai pas eu. Et je ne l'ai peut-être pas réalisé non plus! 
A.B. Tu as évoqué les débuts de ta carrière de journaliste. J'aimerais avoir quelques précisions supplémentaires. Tu as fait, je crois, de la critique musicale avant de faire de la critique littéraire?

G.M. J'ai fait toutes sortes de choses. J'ai commencé à la Tribune de Sherbrooke. Là, j'ai fait un tout petit peu de critique mais je faisais surtout... de tout, les conférences, la police, même un peu les sports; et j'ai fait de cela aussi au Devoir, quand j'y suis entré. II faut bien dire que, au Devoir, à l'époque, nous étions peu de journal istes. II se trouve, oui, que j'ai commencé à faire un peu de critique musicale, de la critique de cinéma - j'en ai fait pas mal — de la critique de théâtre. La seule chose, je pense, que je n'ai jamais faite dans un journal, c'est la chronique financière. Et pour cause!

Pour ce qui est de la critique musicale, je dirai qu'elle m'a donné l'occasion de faire des exercices d'écriture. Quand on parle de musique, il faut inventer. Les mots ne nous sont pas donnés par les œuvres, il faut absolument les trouver tous. J'ai trouvé cet exercice d'écriture extrêmement précieux. Mais j'étais très incompétent par ailleurs!

A.B. Quelles ont été les grandes étapes de ta formation intellectuelle? Quels auteurs ont eu une influence déterminante sur le développement de ta pensée, de tes idées critiques, de tes goûts littéraires?

G.M. J'ai beaucoup de difficulté à répondre à une question comme cellelà parce que je n'ai pas l'impression d'être très bien formé... Si tu veux, je vais te donner une liste de noms propres. Cela fera une salade, mais ça représente assez bien ce qui m'est arrivé. Le premier nom - propre, c'est Bossuet; ensuite, je dirais Claudel, ensuite Camus. Là je parle d'une période assez ancienne, bien sûr.

A.B. Celle de l'adolescence?

G.M. C'était vers la fin de mon cours classique. J'ai découvert Bossuet en philosophie, peut-être même en rhétorique, je ne me souviens pas exactement; j'ai eu une passion tout à coup, j'ai trouvé absolument superbes ces grandes phrases et j'étais très heureux, bien des années plus tard, de découvrir que Kléber Haedens par exemple, dans son Histoire de la littérature française, partageait mon admiration. C'est un peu gênant d'admirer Bossuet, surtout à notre époque...

A.B. Valéry aussi, dans ses Variétés, dit beaucoup de bien de la période de Bossuet.

G.M. Je n'ai pas lu suffisamment Valéry. (Rires.) J'ai relu, il y a un certain nombre d'années, pour des raisons professionnelles, les réflexions de Bossuet sur la comédie. Évidemment, au point de vue des idées, ça ne me convenait pas du tout mais la phrase, la prose de Bossuet m'a enchanté comme auparavant. 
Claudel, c'est venu un peu plus tard. J'en ai lu un peu au collège mais je ne comprenais pas très bien. C'est surtout à l'université... ( $\mathrm{J}$ 'ai fait de petites études universitaires quand je suis arrivé à Montréal). Camus, à peu près en même temps. Ensuite il faudrait parler d'un poète qui est absolument capital pour moi: René Char. Je l'ai découvert à l'université grâce à un professeur que tu as connu également et qui était, à mon avis, un très grand professeur, le Père Ernest Gagnon. Il nous a donné un cours admirable sur la poésie contemporaine. Ce cours m'a fait connaître Char qui, pour moi, demeure un des plus grands. Char et Claudel font un attelage un peu singulier, mais je pense qu'ils peuvent entrer ensemble dans une même expérience.

A.B. De quel Claudel s'agit-il ? du poète ou de l'auteur de pièces de théâtre?

G.M. Eh bien, il y a eu'changement. Au départ ce fut, je pense, le poète. Ensuite l'auteur de pièces de théâtre certainement, et peut-être maintenant, bien que cela n'annule pas le reste, le prosateur. Je trouve que Claudel est l'un des très grands prosateurs de la langue française. Je pense bien sûr d'abord à ses poèmes en prose de Connaissance de l'Est, je pense aussi à ses textes sur la peinture, dont certains sont d'une beauté absolue; à ses textes aussi sur la musique, enfin sur toutes sortes de sujets. Et en particulier je fais une petite enclave pour les textes d'un humour féroce qui continuent de m'enchanter: je pense, par exemple, au Point de vue de Ponce-Pilate où l'aventure du Christ est racontée du point de vue d'un fonctionnaire romain et où on explique ses motifs qui sont, évidemment, très raisonnables. Et aussi je pense au dialogue sur Wagner, que je relis toujours avec ravissement.

A.B. Et du côté de la philosophie, ou de la pensée en général, y a-t-il eu des ouvrages qui t'on marqué? Ceux de Camus peut-être?

G.M. J'hésiterais à placer Camus du côté de la pensée au sens fort, ou du moins de la philosophie. Oui, c'est quelqu'un qui pense, mais moi je dis - c'est une de mes petites idées, anémiques peut-être - qu'on peut penser sans être philosophe. Et je ne crois pas que Camus soit avant tout un philosophe. Sa pensée est un peu étroite, celle du Mythe de Sisyphe par exemple, mais elle est admirablement têtue et absolument honnête. Cela m'a toujours touché. II s'exprime aussi dans une prose qui me touche beaucoup.

II n'y a pas eu beaucoup d'œuvres philosophiques qui m'ont marqué. Je pourrais parler, pour une certaine époque, de Teilhard de Chardin par exemple, mais disons que cette œuvre m'a donné surtout une libération vitale plutôt qu'une insémination intellectuelle, si on peut dire. 
A.B. Et plus tard, au moment où tu étais déjà formé, il y a eu Marshall McLuhan tout de même qui t'a beaucoup intéressé?

G.M. Oui, et dont je ne serais pas prêt non plus à parler comme d'un penseur. D'ailleurs tu sais que les livres de Marshall McLuhan, surtout au début, ont été très mal reçus en France parce que, justement, on n'y retrouvait pas - je ne sais pas si c'est l'expression exacte que j'ai lue - les habitudes d'exposition qui sont celles, traditionnellement, de la pensée philosophique en particulier, et qu'on retrouve même dans les anti-philosophes d'aujourd'hui. Tandis que McLuhan est plutôt une espèce de machine, une machine extraordinaire qui part d'une chose très simple: le médium, c'est le message. Cette chose extraordinaire, en littérature, nous l'agitons depuis fort longtemps! Alfred DesRochers disait: "Le fond, c'est la forme." Et vice versa. Mais Marshall McLuhan a tiré toutes les conséquences pratiques d'une telle formule.

Donc l'effet sur moi de la pensée de McLuhan - et c'est encore Ernest Gagnon qui me l'a fait lire, je tiens à le souligner; c'était un être d'une curiosité extraordinaire - cet effet a été tout simplement de me faire tourner un peu la tête, pour voir des choses que je ne voyais pas auparavant. Ou pour voir les choses autrement. Et je pense que, plus que toutes les lectures de théorie littéraire que j'ai faites, bien sûr depuis que je suis à l'université mais j'en avais tout de même fait un peu auparavant, c'est la lecture de McLuhan qui a fait que je me suis intéressé plus particulièrement à des questions de forme, depuis un certain nombre d'années.

A.B. Mais as-tu été marqué par le thomisme, avec lequel tu as été mis en contact, fatalement, pendant ta jeunesse? Et si oui, as-tu été a amené à prendre des distances par rapport à lui ? Le personnalisme auraitil joué un certain rôle dans cette prise de distance?

G.M. Le thomisme... II faut dire que je n'étais pas, moi, un des étudiants vedettes au grand séminaire, un de ceux qui étaient forts en thomisme ou qui discutaient à perte de.vue là-dessus. Cela m'intéressait, bien sûr, et je dois dire que je conserve un assez bon souvenir de ces études. L'influence du thomisme dans mon cas, ce serait plutôt, peut-être, un certain attrait pour la pensée en général. Une certaine simplicité, parce que saint Thomas a écrit de façon très simple: la preuve en est qu'on peut le lire en latin avec assez de facilité. Mais ce fut une sorte d'éveil de l'intelligence, une formation un peu abstraite de l'intelligence qui, je pense, m'a été très utile. Et surtout, ce qui me frappe c'est que, quand j'ai commencé à lire Camus par exemple, j'ai eu l'impression que le thomisme, ou du moins les lectures que j'avais faites à l'intérieur de ce domaine, m'étaient extrêmement utiles pour entrer dans Camus. Je n'ai pas senti de hiatus profond. Les questions fondamentales que j'avais rencontrées dans le thomisme se pré- 
sentaient chez Camus et, disons, avec le même degré de gravité ou d'importance.

\section{A.B. Mais tu as fait l'économie de Maritain et de Mounier?}

G.M. Je n'ai pas fait l'économie de Maritain. Je l'ai lu, Maritain, et j'ai encore de l'affection pour certains de ses livres, en particulier ses Sept leçons sur l'être, un livre de proses philosophiques - tu remarqueras l'expression - que j'ai toujours trouvé très beau. Bien sûr, le livre de Maritain qui a marqué à l'époque, c'était Humanisme intégral. Et ce livre représentait surtout - c'était un des problèmes majeurs de ce temps - une rupture dans les manières de considérer les rapports de l'Église et de l'État. C'était vraiment une libération. Maritain décidait de prendre ses distances par rapport à certaines formes de chrétienté, de collusion de l'Église et de l'État. Ce livre a été très important en France et ici, beaucoup plus important qu'on ne peut l'imaginer maintenant. Son effort a été poursuivi, bien sûr, par Mounier et par toute l'équipe d'Esprit. Ce qu'a fait Mounier principalement, je pense, c'est de,permettre une libération, sur plusieurs plans mais en particulier sur le plan politique, de la pensée je ne dirai pas "chrétienne», mais de la pensée des chrétiens.

A.B. Cela m'amène à t'interroger sur la Relève. Les écrivains de cette revue accordaient beaucoup d'importance à la pensée d'un Maritain. As-tu été en contact assez tôt avec la pensée de l'équipe de la Relève? Lisais-tu la revue quand tu étais aux études?

G.M. Ah non! J'ai lu la Relève très tard, bien après la disparition de la revue. Quand j'étais étudiant, quelles revues lisions-nous? J'essaie de le retrouver... Il y avait l'Action nationale, plutôt à droite, bien sûr; il y avait peut-être Relations, qui n'était pas à l'extrême-gauche... mais je ne me souviens pas d'avoir vu la Relève, ou la Nouvelle Relève, avant mon arrivée à Montréal. Et même quand je suis arrivé à Montréal, à la fin des années quarante, je n'ai pas lu la Relève encore à ce moment. En fait, j'ai connu des gens de l'équipe bien avant de m'intéresser à la revue. J'ai connu d'abord Jean LeMoyne, à l'occasion d'un article que j'avais écrit sur Saint-Denys Garneau, au tout début des années cinquante; puis j'ai fini par connaître tous les autres. Je suis entré un peu dans le groupe, qui n'était plus le groupe de la revue mais un groupe d'amis - Robert Élie, Louis-Marcel Raymond... Jean Simard s'y est joint lui aussi. C'était, je dois le dire, un groupe extraordinaire. Ce qui m'a fasciné, dans ce groupe, c'était d'abord la générosité intellectuelle. J'ai été accueilli, chez ces gens qui étaient mes aînés, avec une liberté extraordinaire, et bien sûr, le thème premier des conversations et des discussions, c'était ce dont je parlais tout à l'heure à propos de Maritain et de Mounier c'est-à-dire une libération de la pensée chrétienne (disons-le, cette fois). Aussi, ce qui m'a beaucoup frappé chez eux, c'est la profondeur de la culture. Ce sont des gens 
qui avaient, qui ont, une culture profonde, une culture de relecture, une culture d'attention extrême, et qui étaient en rapport vraiment constant avec la vie. On a parfois parlé des intellectuels de la Relève et de la Nouvelle Relève comme de gens assez abstraits et qui étaient assez éloignés des questions...

A.B. ... sociales?

G.M. Oui... D'abord ils n'étaient pas éloignés des questions sociales, comme on l'a dit; ils ne l'étaient certainement pas au moment où je les ai connus. Ce qui me frappe surtout donc, c'était que tout, chez eux, communiquait. Nous avions des discussions absolument incroyables et longues sur les derniers Quatuors de Beethoven, par exemple, sur des œuvres littéraires, et je me souviens que, un an avant sa mort, je pense, un homme comme Robert Élie me disait qu'il venait de relire tout Rimbaud et qu'il croyait avoir compris la raison de son abandon de la poésie. Je regretterai toujours de ne l'avoir pas interrogé plus avant sur ce thème; mais je cite cet exemple pour montrer comment, pour Robert Élie, Rimbaud était quelque chose d'essentiellement vivant, qu'il a porté avec lui toute sa vie. Et on pourrait en dire autant d'autres passions de lecture, chez d'autres membres du groupe. Je dois ajouter que moi, j'admirais immensément, j'ai beaucoup profité de cela mais je suis toujours demeuré un petit peu... pas envieux, non, ce ne serait pas le mot, mais j'ai toujours eu l'impression de n'être pas tout à fait au niveau de ces intellectuels, qui avaient ce que j'appelais tout à l'heure cette culture profonde, qui était extrêmement riche.

A.B. Te sentais-tu plus près de la problématique de cette génération que de celle de l'Hexagone et de Liberté?

G.M. Pas facile à dire. Je pense que, au départ, la problématique des écrivains de l'Hexagone n'était pas fondamentalement différente de celle de la Relève. Je ne parlerai pas de Cité libre parce que Cité libre s'est intéressée assez peu à la littérature. Je pense qu'il s'agissait toujours, dans les deux groupes, et malgré les différences de génération qui imposaient des différences de ton, etc., il s'agissait toujours d'arriver, par la parole, à l'expression et à la libération de la vie. Et on pourrait dire, je pense, dans les deux cas: de la vie intérieure. Si on pense aux premières publications de l'Hexagone, celles qui s'étendent sur les six ou sept premières années, on peut trouver beaucoup de poèmes qui ne sont pas éloignés de ce que, dans l'intention profonde, on pouvait imaginer à la Relève. La différence peut-être principale, c'est que, à l'Hexagone, on n'était pas philosophe du tout, on n'était pas métaphysicien pour deux sous, alors que, à la Relève, il y avait cette inquiétude métaphysique, cette inquiétude religieuse et philosophique qui était très forte.

A.B. Tu dis que à l'Hexagone, on n'était pas philosophe. II faudrait ajouter que le sentiment religieux y est beaucoup moins présent que dans la 
génération de la Relève. Est-ce que la génération de l'Hexagone n'a pas rejeté la religion?

G.M. Plus tard, peut-être; mais au début, ce n'était pas sensible du tout. Enfin, je n'ai pas été très près de l'équipe de l'Hexagone. J'ai connu Miron, à l'époque, j'en ai connu d'autres; j'étais celui qui recevait leurs poèmes au Devoir; je les ai beaucoup publiés d'ailleurs dans les pages du Devoir (on y publiait des poèmes, à cette époque) et j'écrivais des articles sur eux. Je pense que l'Hexagone au départ n'a pas eu une attitude anti-religieuse, certainement pas, et même pas areligieuse. Dans les premières publications de l'Hexagone l'inquiétude religieuse est moins virulente que chez les aînés mais...

A.B. Elle est tout à fait essentielle, cette inquiétude religieuse, dans la problématique de la Relève. À l'Hexagone, elle me semble beaucoup moins marquée.

G.M. Oui, bien sûr. Elle est moins marquée, mais il faut bien dire que, à l'Hexagone, on n'avait pas de prosateurs. À la Relève, on écrivait des articles, on agitait des questions. À l'Hexagone on faisait des poèmes, et le poème n'est pas le lieu d'une expression directe de ces choses-là. Même les poèmes de Saint-Denys Garneau, quand on y pense, il y en a très peu qui ont des connotations relig ieuses assez évidentes. Je ne verrais surtout pas une rupture, à ce moment-là. La rupture se produira peut-être plus tard. Tu parlais de Liberté tout à l'heure: il y aura, à Liberté, des prises de position qui seront en rupture par rapport à certaines des positions de la Relève.

A.B. Et comme romancier, tu sens-tu plus près d'un Robert Élie que d'un Hubert Aquin? ou est-ce le contraire?

G.M. J'ai beaucoup de difficulté à me situer comme romancier parce que j'ai beaucoup de difficulté à penser à moi comme romancier, et c'est une question qui ne m'est jamais venu à l'esprit. À vrai dire, je me sens très éloigné des deux: à la fois de Robert Élie et de Hubert Aquin. Pour ce qui est du premier, je me souviens que La Fin des songes, son premier roman et celui qui a obtenu le succès le plus considérable, m'avait paru très obscur. J'avais beaucoup de difficulté à entrer dans ce roman. Bien que, peut-être, on puisse découvrir entre mon premier roman à moi et la Fin des songes certaines parentés; pour moi, au niveau de la sensibilité - et en tant qu'auteur, je parle surtout à ce niveau - le rapport n'est pas très net. Quant à Hubert Aquin, pour moi c'est un monde assez étranger. Ce n'est pas vraiment une des cuvres de la modernité québécoise qui me touche le plus. Là où je me sens extrêmement à l'aise, c'est, bien sûr, chez Ducharme, au premier chef. II y a des œuvres que j'admire à part cela. Je vais encore faire la liste, comme je l'ai fait tout à l'heure pour les auteurs qui m'ont influencé. II y a Jacques Ferron, parmi les plus anciens. Je me suis toujours trouvé à l'aise chez lui; il y a une allé- 
gresse, une liberté de la langue, chez Ferron, qui compense abondamment les défauts majeurs que son œuvre présente. Ensuite je parlerais de Bessette, qui est un écrivain extrêmement différent - Monseigneur Bessette de Kingston, comme dit Ferron - mais dont l'œuvre m'importe beaucoup, peut-être parce qu'il y a là une passion pour le réalisme, une volonté de réalisme, qui est contrariée mais de façon féconde. Sans doute y a-t-il toujours eu chez moi le rêve de faire un roman réaliste, ce pour quoi d'ailleurs je ne suis pas doué du tout. Ensuite, je reviendrais à d'autres écrivains plus modernes comme Réjean Ducharme, bien sûr. Réjean Ducharme, j'ai lu chacun de ses romans plusieurs fois et là, je me sens très à l'aise; et j'attache aussi une grande importance à Jacques Poulin.

Ce sont des auteurs très différents de moi. C'est peut-être une donnée de ma nature, d'aimer surtout des œuvres qui ne me ressemblent pas.

\section{B - Les romans}

A.B. Avant que nous passions tes romans en revue, j'aimerais te poser quelques questions sur l'ensemble. Et d'abord sur un thème assez important, je crois, celui de la banalité. Dans ton dernier ouvrage, La Littérature et le reste, tu affirmes: « Peut-être au fond n'ai-je exprimé que cela dans mes trois petits romans, une véritable passion du banal. " II semble en effet - c'est explicite dans le Poids de Dieu que la vie, au sens fort, se trouve du côté du quotidien, des joies et des malheurs communs, ceux de tout le monde. Est-ce bien ton avis? Et comment justifies-tu. cette position, toi qui, d'autre part, es un passionné de Rimbaud et de Claudel, bref de poètes qui sont tout le contraire de chantres du quotidien? Et puis, la passion du banal, n'est-ce pas une contradiction dans les termes, la passion transfigurant toujours son objet en quelque chose d'exceptionnel?

G.M. Je commence par répondre à ta dernière observation. En effet, l'expression «passion du banal» peut paraitre contradictoire. Mais je pense qu'il y a, dans le banal, beaucoup de passion. J'oppose banal à exceptionnel, et quand je pense à exceptionnel, je pense à héros, je pense à des romans où il y a des actions, donc, exceptionnelles. Mais le banal et le passionné ne s'excluent pas nécessairement. On n'a qu'à regarder autour de soi. Dans la vie même, sinon dans l'écrit - c'est peut-être un peu différent, on y reviendra - je pense qu'il y a des passions extrêmement violentes qui s'expriment sous les apparences, justement, du banal. Ce qui voudrait dire que le banal n'est jamais banal... et c'est bien ce que je pense! Quant à mes lectures tu as parlé de Rimbaud et de Claudel - bien sûr, ce sont des auteurs qui ont pratiqué l'excès, l'abondance. Chez Claudel, on est dans l'énorme, et chez Rimbaud, on est dans tous les excès. Mais on peut 
les lire aussi, d'une certaine façon, en fonction du banal. Et là, je pense surtout à Une saison en enfer, de Rimbaud. Ce qui me frappe dans ce poème, c'est que cette éruption extrêmement violente de langage se fait à partir des éléments les plus ordinaires, les plus banals, à partir de la culture parfaitement scolaire d'un jeune homme qui était un premier de classe. De quoi part-on dans Une saison en enfer? On part du petit catéchisme; la figuration, dans le texte, est très proche du petit catéchisme en images. On peut imaginer les illustrations de Gustave Doré. L'autre source, c'est l'histoire de France, c'est Nos ancêtres les Gaulois, c'est l'histoire de France la plus simple, celle qu'on trouve dans les manuels. Et c'est à partir de tels éléments, c'est à l'intérieur de ces textes-là que Rimbaud travaille. Quant à Claudel, il y a chez lui des pages absolument extraordinaires sur le banal. Je pense à La Messe là-bas, je pense aux Feuilles de saints où il présente les saints comme des gens qui, justement, sont pris dans le courant de la vie plus ordinaire. Tu me diras, bien sûr, et je te donnerai raison, que c'est l'expression qui compte, chez ces auteurs, et que leur expression s'éloigne immensément de la banalité. Là-dessus, je te répondrai ce que j'ai déjà dit tout à l'heure: j'ai précisément besoin d'auteurs qui ne me ressemblent pas, qui me dépassent, qui m'entraînent où je ne vais pas de moi-même et qui expriment un besoin de grandeur, de révolte peut-être, d'excès que je n'accomplis pas dans ma vie ou dans mes écritures. La lecture pour moi, c'est aussi, et c'est peut-être surtout, une expérience de l'altérité. Et les livres, souvent, qui m'enchantent sont les plus éloignés de mes manières personnelles.

A.B. Mais qu'est-ce qui t'intéresse dans le quotidien et dans le banal? Qu'est-ce qui en fait le prix pour toi?

G.M. C'est lié, d'une certaine façon, à une conception de la vies sans doute. Pour moi, l'essentiel se passe dans les circonstances les plus ordinaires de l'existence. Et si on quitte ces circonstances, je pense qu'on est toujours traître par rapport à ce qui fait l'essentiel de sa propre existence. Je conçois qu'on puisse penser différemment, rêver d'héroïsme, et j'irais même plus loin: peut-être ce refus de l'héroïsme chez moi provient-il d'une certaine timidité, d'une certaine peur. Ce n'est pas impossible du tout. Mais enfin, je pense qu'il y a, dans ce banal qui m'intéresse, qui me passionne, une certaine vérité, qui est d'ailleurs liée à une conception chrétienne du monde selon laquelle tout être humain, quel qu'il soit - l'intellectuel, l'écrivain, ou la personne qui ne s'exprime pas - a, au regard de Dieu (disons-le) une importance égale, et très considérable. Tout être humain est aimé pour ce qu'il est, il est précieux en lui-même. D'autre part, ma passion pour le banal a sans doute des raisons plus exactement littéraires. Le banal, pour moi, est lié à une certaine forme de prose. Ce que je cherche dans la prose, c'est l'uni, le continu, une sorte de mouvement qui comporte des modulations, bien sûr, mais qui ne s'éloignent jamais beau- 
coup de la ligne principale. Il y a donc une convenance entre le genre de prose dont je rêve, et que je trouve parfois chez Camus, et une thématique du quotidien.

A.B. Ce refus des actions extérieures éclatantes n'est-il pas aussi un façon de valoriser les événements de la vie intérieure? L'exaltation du quotidien ne favorise-t-elle pas l'attention à ce qui se passe dans les profondeurs mêmes de l'être?

G.M. Tu as sans doute raison, bien que j'aie une certaine difficulté à m'aventurer sur ce terrain. La vie intérieure, je m'en méfie un peu. II y a une certaine exaltation de la vie intérieure, par opposition à la vie extérieure, à la vie de l'action, à laquelle je ne crois pas. Si la vie intérieure ne correspond pas très exactement à ce qui se passe dans les actions, dans les gestes, je pense que cela devient une sorte de caisse de résonance, et rien que cela. Ce qu'on appelle la vie intérieure, ça peut être un superbe vide. D'un autre côté, il faut bien dire que, - en faisant toujours la distinction entre l'écriture et la vie, pour moi ce n'est pas la même chose, même si c'est lié très profondément -, je ne suis pas très doué pour l'action. J'ai toujours rêvé d'écrire un roman policier, mais pour cela il faudrait inventer une action, pouvoir imaginer facilement des personnages divers, colorés, etc., imaginer aussi une intrigue qui se tiendrait bien et je t'avoue que, sur ce plan-là, je me sens plutôt démuni.

A.B. Dans tes trois romans, un même scénario fondamental est à l'œuvre: le personnage est d'abord plein d'assurance, il trouve son plaisir dans une action qui le met en rapport avec le monde concret, ordinaire une action donc qui n'a pas le côté fastueux de celles du roman policier, mais une action tout de même. Puis le personnage trouve son chemin de Damas. Ses belles certitudes sont ébranlées, il sombre dans l'angoisse et, chaque fois, c'est l'amour qui est à l'origine de la crise. Dans le Poids de Dieu, bien sûr, ce n'est pas l'abbé Savoie qui devient amoureux mais, tout de même, la bénédiction (ratée) des fiançailles de Serge Normand, en qui il se projette, est l'événement qui déclenche la crise. Maurice Parenteau va rater, lui aussi, son mariage, et l'amour l'aura fortement secoué, tout au long du roman. Qu'estce qui, dans l'amour, est perturbateur à ce point? L'amour d'ailleurs n'est-il pas avant tout, dans tes romans, ce qui dérange, ses aspects négatifs ne sont-ils pas plus marqués que les positifs (pouvoir d'enchantement, etc.)?

G.M. Ce que tu dis est très juste, surtout à la fin. Je ne suis évidemment pas un écrivain de l'exaltation amoureuse, du bonheur amoureux. Pour moi, en effet, dans le roman, l'amour est essentiellement ce qui dérange, ce qui brise, ce qui emporte, ce qui conduit là où on ne veut pas aller. On pourrait reparler, à ce propos, de Claudel.

A.B. Dans Partage de midi? 
G.M. Dans Partage de midi, bien sûr, mais aussi dans l'ensemble de son ceuvre. Pour Claudel, l'amour est une immense déchirure, une blessure qui ne se referme jamais et qui est donc, toujours, une voie d'accès à autre chose. Ne parlons pas de l'infini; disons simplement: autre chose. C'est ce qui se produit aussi, ou qui tente de se produire dans mes romans. Mes personnages sont des gens qui ont de la difficulté à aimer. Et là ce n'est peut-être pas claudélien, c'est... J'ai des personnages qui sont plutôt fermés sur eux-mêmes, plutôt solitaires. Chez Parenteau, bien sûr, la fermeture prend un aspect plus volontaire que chez les autres mais il y a là, quand même, un trait commun aux trois personnages. Ils ont de la difficulté à aimer, parfois ils s'y refusent, et tout à coup ils tombent dans l'amour comme dans un véritable piège. Cette aventure fait qu'ils sont plus ouverts, à la fin du récit - même Parenteau - qu'ils ne l'étaient au début. C'est tout ce que je leur demande d'ailleurs! Pour moi, l'aventure d'un personnage est essentiellement une expérience d'ouverture.

A.B. Mais alors l'amour est un peu cette perturbation qui vient des profondeurs, et qui arrache l'être à ce fonctionnement en société qui, au départ, le satisfait complètement. N'y a-t-il pas là une opposition entre extérieur et intérieur, et peut-être même entre l'action - une action coutumière, répétitive - et ce qu'on pourrait appeler la vie intérieure?

G.M. II y a une opposition, oui, dans un certain sens. Tu parles de satisfaction complète dans l'action. Je pense que ce n'est pas tout à fait juste. Mon petit curé du Poids de Dieu, dès le départ, est un peu inquiet, il n'est pas tout à fait accordé à son action. En fait, je pense qu'il rêve de l'action comme d'une solution, mais la solution ne peut pas être dans l'action. Pour ce qui est de Parenteau, je pense que lui, il en met vraiment un peu trop! Ce trop masque un moins, c'està-dire une confiance très limitée dans l'action. D'autre part le dernier, Marcel Fournier, est un méditatif qui est arraché à sa méditation, à ses travaux universitaires, à ses réflexions, par l'action qu'il doit mener comme fonctionnaire. Donc l'action, à mon avis, ne les satisfait pas entièrement; ils sont dans une espèce de perplexité profonde. Ça me semble bizarre de raisonner comme cela sur des personnages, surtout sur les miens...) II y a désaccord profond entre leur désir et leur action. Ou plutôt, accord incomplet; parce que, s'il y avait un désaccord violent, il ne s'agirait pas d'un de mes romans! Et l'amour, c'est la déchirure, mais la déchirure qui implique une perspective de réunion avec soi-même.

A.B. Dans La littérature et le reste, tu affirmes que tes trois héros sont le prêtre ordinaire, le journaliste ordinaire, le fonctionnaire ordinaire (je reviens à cette question du banal, ou du quotidien, dont nous parlions tout à l'heure). Il me semble cependant que l'affirmation n'est pas tout à fait exacte car, tous trois, tes personnages détiennent une autorité, 
exercent un pouvoir. L'abbé Savoie, du fait de sa prêtrise, est au-dessus des autres hommes, d'une part; d'autre part, il est aumônier d'un syndicat et sa parole compte - plus, sans doute, qu'il ne voudrait! Maurice Parenteau est directeur de son journal et Marcel Fournier est un haut fonctionnaire. Tes héros ne sont-ils pas au-dessus du commun?

G.M. Je dois répondre d'abord que mes.personnages devaient être choisis dans des métiers que je connaissais un peu. Il se trouve que j'ai pratiqué des métiers de cet ordre-là.

Deuxième remarque: ce sont des gens qui, dans le cours du roman, ou vont se désister volontairement du pouvoir, ou vont lutter contre lui. Je pense que c'est vrai de Claude Savoie et de Marcel Fournier: ces gens sont en situation de pouvoir mais refusent de l'exercer. Quant à Maurice Parenteau, il sera privé du pouvoir, finalement, par des circonstances apparemment extérieures, mais le résultat sera le même. L'idée de pouvoir, pour moi - je ne sais pas si je dois employer ce mot de pouvoir pour mes personnages, mais employonsle - elle est liée à celle de responsabilité. Or la responsabilité et le banal sont liés de façon assez profonde. Chaque être dans la vie; quelle que soit la circonstance où il se trouve - là je devais trouver des circonstances qui mettaient mes personnages un peu, comme tu dis, au-dessus du commun, mais ce n'est pas l'important - , chaque être est responsable par rapport aux autres. Et cela, dans les circonstances les plus courantes de l'existence. Ce que j'ai probablement voulu exprimer à travers mes personnages, c'est cette responsabilité à l'égard des autres. Je dois dire que, dans la vie, rien ne me scandalise plus que l'abus de pouvoir. Que des curés, que des fonctionnaires, que des journalistes, et la liste pourrait s'allonger indéfiniment, utilisent ce pouvoir (oui, ils en ont un) sans en faire un service.

A.B. Poursuivons sur ces héros qui sont un prêtre, un journaliste et un fonctionnaire. N'y a-t-il pas une gradation entre eux: leur rapport à la collectivité n'est-il pas de plus en plus anonyme, indifférencié ? Pour le prêtre, tous les êtres sont, virtuellement, des Serge Normand c'està-dire des personnes possibles. Le directeur de journal s'intéresse, lui, à des individus - par exemple le maire, suspect d'escroquerie. Et le fonctionnaire perd de vue toute dimension individuelle. En somme, est-ce que la dimension d'intériorité, en tant que liée à la fonction sociale du personnage, n'est pas progressivement évacuée?

G.M. Oui, c'est très juste. II y aurait moyen de faire un peu de sociologie, à partir de l'observation que tu fais. L'évolution que tu vois, d'un personnage à l'autre, correspond dans les grandes lignes à ce qui s'est passé dans le domaine social. A la fin des années cinquante, quand j'ai écrit /e Poids de Dieu, la personne était, en effet, au centre des préoccupations. Cette considération de la personne dans notre 
milieu - mais on pourrait aussi inclure, dans cette observation, des ensembles beaucoup plus vastes - est devenue beaucoup plus difficile. Dans cette perspective, le retrait dans la vie intérieure d'un Marcel Fournier est un peu inquiétant. II est profondément ambivalent. Je pense qu'il y a chez ce personnage, dans la mesure où je peux en parler comme d'un être réellement indépendant, une tentative réelle de se ressaisir dans le tohu-bohu, dans le vertige de l'existence, mais il y a peut-être aussi le risque du vide. Ce qu'il cherche, dans ce que tu appelles sa vie intérieure, c'est peut-être un cul-de-sac, un refuge par rapport à ce qui se passe réellement, et c'est peut-être même un aveu d'impuissance à réaliser la personne.

A.B. Tu disais tout à l'heure que l'exercice de l'autorité, il fallait le concevoir comme un service. N'est-ce pas beaucoup plus difficile justement pour un Marcel Fournier qui est fonctionnaire, bref pour quelqu'un qui agit dans le cadre d'une société fortement bureaucratisée? La notion de service n'est-elle pas liée à celle de personne?

G.M. Si tu permets, je répondrai à cette question par un seul mot: oui! (Rires.)

A.B. Parlons maintenant du Poids de Dieu. Je voudrais t'interroger sur ce que Genette appelle la voix narrative. Le roman est d'abord un récit à la troisième personne, strictement focalisé (sauf en de rares passages) sur le personnage principal, Claude Savoie, qui est un IL à valeur de JE; puis ce IL devient JE, le récit se fait à la première personne. Cette transformation de la voix narrative est sans doute en rapport avec l'évolution de la problématique du roman? Comment la vois-tu, cette évolution?

G.M. J'aurais deux observations à faire là-dessus. D'abord, comme je te l'ai dit précédemment, j'ai commencé ce roman non pas comme un roman mais comme une sorte d'expérience d'écriture, à des fins personnelles. Et il était nécessaire pour moi d'employer la troisième personne, pour éviter une sorte de confusion, pour objectiver, dans une certaine mesure, ce que j'essayais de raconter, d'éclairer. Je suis passé au JE à la fin et je pense que, en effet, cela devenait nécessaire parce que ce IL, au fond, comme tu dis, n'était qu'un JE différé. Quand même, il y avait une difficulté d'arriver au JE c'est-à-dire d'effectuer la symbiose, en quelque sorte, du narrateur et de son personnage, ou de l'auteur si tu veux. Et cela ne pouvait se faire qu'à partir du moment où le personnage avait atteint une certaine libération. D'un autre côté, je dois dire - c'est anecdotique, mais peut-être pas sans intérêt - que la dernière partie du roman a été écrite non pas ici mais en France, au cours d'un séjour extrêmement heureux où je me suis senti beaucoup plus libre. Cela m'a probablement permis d'utiliser, de poser un JE que je n'aurais peut-être pas pu poser si j'étais resté ici. 
A.B. Mais toutes ces raisons me semblent passablement extérieures au roman. Le passage au JE n'est-il pas lié à la logique même du récit?

G.M. Oui, il l'est, et c'est impliqué dans les remarques que je viens de faire. Voici un personnage qui, étant appelé IL, non seulement est maintenu par le narrateur dans une certaine distance, mais se maintient luimême à distance par rapport à sa propre personne. Il ne coïncide pas tout à fait avec lui-même: tel est le sens qu'on peut donner à l'emploi de la troisième personne. Il y $a$, dans la dernière partie, dirais-je prudemment, une amorce de prise de conscience qui est exprimée par le JE.

A.B. Dans le Poids de Dieu, la révolte est un thème important. Claude Savoie se révolte contre l'institution cléricale à laquelle il appartient et qu'il n'avait jamais interrogée vraiment, jusque-là. Par ailleurs, le personnage semble bien accordé aux tâches pratiques du ministère (il refuse même de trouver refuge dans l'enseignement). Comment comprendre alors la révolte de Savoie? Contre quoi est-elle dirigée exactement? Le personnage, partagé entre son goût pour l'action et un goût égal pour la vie intérieure, n'est-il pas trop équilibré, en somme, pour connaître une révolte profonde?

G.M. J'aime bien l'expression que tu emploies: “trop équilibré pour connaître une révolte profonde». Cependant je ne suis pas sûr qu'il s'agisse d'un équilibre. Je pourrais parler peut-être de deux forces contradictoires qui s'exercent en lui, qui le tirent à hue et à dia et qui empêchent peut-être, justement, la révolte de s'exercer. S'il y a révolte chez Claude Savoie, j'ai l'impression qu'elle s'exerce avant tout, bien que ce ne soit pas dit dans le roman de façon explicite, contre luimême. Il est fâché contre l'Église, si l'on veut, mais je pense qu'il est encore plus profondément fâché contre la façon dont il vit lui-même l'Église. L'Église institution est une sorte de projection qu'il fait de ses propres empêchements. C'est ainsi que je lirais aujourd'hui. Je serais curieux de savoir si un autre lecteur peut le lire de la même façon. Il est bien sûr que la révolte de Claude Savoie, il est assez difficile d'en définir les objets précisément, même si on parle de l'Église, parce que tout cela est un peu en porte-à-faux.

A.B. Le Poids de Dieu pose aussi le problème de la difficile conciliation entre les intérêts de Dieu, défendus par l'Église, et ceux de l'homme, ou plutôt des hommes concrets, auxquels l'Église reste trop souvent étrangère. Pierre Dupas, dans le Temps des hommes de Langevin, vivait aussi ce conflit, et sa recherche de solution débouchait sur un échec. Toi, au contraire, tu trouves une solution, qui s'exprime par l'Introïbo final. Pourrais-tu l'expliciter?

G.M. Pas facile! - D'abord, la revendication du droit des hommes par rapport au droit ecclésiastique, je dirais presque le droit canon, correspond à ce que j'ai dit tout à l'heure à propos du banal, du quotidien, 
de l'homme comme valeur quasi absolue. Je dis bien "quasi „... cela ouvrirait une série de discussions qui seraient assez importantes et assez difficiles. Pour ce qui est de la solution dont tu parles, je dois dire qu'on me l'a reprochée. Je me souviens de conversations que j'ai eues à l'époque, notamment avec un prêtre qui me disait que, ce que j'aurais dû faire, c'est le suicide de Claude Savoie; et que j'avais peut-être manqué un peu de courage. Cela, bien sûr, c'est possible. D'autre part, j'ai de la difficulté à voir une "solution", comme tu le dis, dans l'Introïbo final. II est important de noter que Introïbo, cela s'écrit au futur. Cela traduit le verbe entrer, sans définir dans quoi on va entrer. Je vois le personnage, à la fin du roman, comme étant essentiellement en disponibilité. II a eu sa crise qui a été, pour moi, extrêmement violente. Il a été très profondément secoué, et il refuse, à la fin, de dire ce qu'il sera. II se dit tout simplement disponible. À quoi? Tu te souviendras que j'ai cité Camus au début du dernier chapitre. Il est disponible à ce que Camus exprime, je pense, de plus profond c'est-à-dire l'acceptation de la vie. La vie telle qu'il en parle dans Noces par exemple, et qui est quelque chose de... (c'est banal "effrayant" ce que je dis-là!). Enfin c'est un homme qui... - Tout ce qu'il refuse de lâcher, c'est l'espérance! Et il vient de découvrir aussi qu'il pourrait être incroyant. Sa grande libération, c'est que sa foi ne s'impose plus à lui comme une fatalité; que sa foi pourrait, qu'elle pourra - employons tous les temps verbaux qui pourraient convenir - se produire dans une liberté.

A.B. Elle devient donc un choix libre. Mais que fais-tu de la grâce, à ce moment? Peut-on choisir librement d'avoir la foi ?

G.M. Tu me parles comme un janséniste. (Rires.) Je n'aime pas beaucoup parler de la grâce. Je viens de terminer la lecture de la biographie de Mauriac par Jean Lacouture, livre qui m'a passionné, et bien sûr, Mauriac parle beaucoup de la grâce; mais Mauriac était très janséniste par certains côtés. II ne l'était pas complètement... Mais cette conception de la grâce qui nous tombe dessus, venant on ne sait d'où, par quels chemins - comme une espèce de fatalité, justement -, moi je n'y crois pas.

A.B. Parlons maintenant de ton deuxième roman, Retour à Coolbrook. Dans ce livre, un rapport étroit s'établit entre la ville et la femme: Lucile, c'est Montréal; Mariette Sainval, c'est Coolbrook (la ville natale de Maurice Parenteau). La présentation en page 4 de la couverture suggère que l'histoire d'amour entre Maurice et Mariette aurait quelque chose de superficiel par rapport à l'histoire d'amour entre Maurice et sa ville natale. Qu'en est-il exactement? Que représente le rapport à la ville, en regard du rapport à la femme aimée et, peut-être, dans l'inconscient du personnage, en regard du rapport à la mère?

G.M. La question de la ville est évidemment essentielle. La ville natale... Tu m'embêtes un peu avec cette histoire d'inconscient, de rapport à 
la mère, mais enfin, oui, j'y vais, je me compromets, je plonge. Tu as raison, la ville dans cette histoire est bien maternelle, et l'aventure de Parenteau constitue, en quelque sorte, une régression. II retourne à Coolbrook comme on retourne - fantasmatiquement bien sûr — dans le sein maternel. Et puis, tiens, j'y pense - cette idée ne m'était jamais venue auparavant, c'est ta question qui la suscite -, c'est la raison pour laquelle le nom de Maurice Parenteau, son nom propre, n'apparaît qu'à la dernière ligne du roman. Il reçoit un nom parce que, justement, il vient de naître; il est retourné dans le sein maternel, puis il en est expulsé, il naît vraiment, à la fin du roman il est tout seul, tout nu, comme un bébé. Pas étonnant que la belle Mariette ait épousé quelqu'un d'autre. Elle jouait un rôle assez trouble dans l'histoire. Pas étonnant, non plus, que le narrateur ait de la difficulté, à la mettre au foyer: elle n'est jamais ce qu'elle devrait être, ce qu'elle pourrait être pour lui.

A.B. Le rapport de Maurice Parenteau à Lucile me semble beaucoup plus superficiel que son rapport à Mariette Sainval. À ce moment, le défi que représente la grande ville, Montréal, n'est-il pas un défi tout extérieur par rapport à celui que représente Coolbrook?

G.M. Je dirais plus justement que c'est le défi de l'extérieur. Le défi de l'altérité. En allant vers Mariette Sainval comme vers Coolbrook, Maurice Parenteau va, dans une certaine mesure, vers le même. Tandis que Lucile, c'est l'autre, et c'est peut-être pour cela d'ailleurs qu'elle paraît superficielle. Mais je ne suis pas sûr que le rapport entre Parenteau et Lucile soit superficiel. On voit, il me semble que c'est assez net dans l'écriture, dans le récit même, que Parenteau fait tous les efforts pour que ce soit un rapport superficiel et pour écarter ce défi qui est contraire à son projet premier, qui est un projet de régression. II s'en va à Coolbrook pour s'éteindre lui-même, pour retourner à une espèce d'origine qui est le lieu de l'indifférenciation. Tandis que Lucile, c'est la différence, et Montréal c'est la différence par rapport à Coolbrook, c'est aussi le lieu de toutes les différences. Voilà pourquoi Parenteau fuit ce lieu, d'une façon extrêmement décidée!

A.B. Maurice Parenteau, contrairement à Claude Savoie, est un incroyant et, comme il est le narrateur, il exprime une attitude qui est le contraire de la tienne sans que la contrepartie apparaisse dans le roman. Cela ne t'a-t-il pas gêné même si, comme romancier, tu entends laisser à ton personnage toute liberté? Et pourquoi avoir choisi un personnage d'incroyant? D'autre part, la religion ne joue-t-elle pas secrètement un rôle dans le récit? N'est-ce pas tout de suite après la messe de minuit que commence le récit de la rupture, comme si l'incroyance de Pareanteau trouvait là son châtiment?

G.M. II y a beaucoup de choses dans ta question et elles sont presque toutes redoutables! Je dirai d'abord que Parenteau n'est pas un personnage libre. Il est entièrement enchaîné - je parle, évidemment, d'un 
point de vue subjectif - à moi-même. Et je ne suis pas sûr qu'il exprime une attitude contraire à la mienne. J'ai signalé tout à l'heure que Claude Savoie, à la fin du Poids de Dieu, découvrait qu'il pouvait être croyant parce qu'il pourrait être incroyant. Parenteau, c'est la part d'incroyance qu'il y a en moi. Je pense qu'elle est extrêmement vivace, et elle l'est aussi chez tout croyant. Parenteau n'est donc pas le contraire de ce que je suis. Il est une partie de moi-même. J'ai commencéà écrireRetourà Coolbrook, justement, toutdesuiteaprèavoir fini Le Poids de Dieu. J'avais besoin de cette espèce de contrepoids, de l'expérience très différente qu'impliquait ce personnage, et je t'avoue qu'il ne m'a jamais gêné. Je me suis toujours senti extrêmement à l'aise avec Parenteau, et c'est peut-être celui de mes personnages avec lequel j'entretiens les rapports les plus amicaux.

A.B. Y a-t-il un lien entre l'incroyance de Parenteau et la régression qu'il vit? La première est-elle le signe de la seconde?

G.M. On pourrait dire exactement le contraire! Tu signales cette histoire de la messe de minuit. (Dans laquelle tu vois un châtiment?... Pas moi ; c'est entièrement étranger à ma perspective. Enfin, c'est peut-être toi qui as raison: c'est toi qui es le lecteur...) Parenteau revient à sa ville natale et il revient, au cours de la messe de minuit, à sa religion natale; et on pourrait dire que le retour à la religion implique une régression, qu'il est peut-être le fond même de la régression pour $\mathrm{Pa}$ renteau. C'est la raison de la rupture quai se produit presque tout de suite après: la régression est accompagnée de l'interdiction, et suivie tout aussitôt de l'expulsion.

A.B. Parlons maintenant de ton dernier roman, ou plutôt de ton récit, intitulé Un voyage. On y trouve une sorte d'art poétique. Le héros du livre réfléchit sur le roman policier dans les termes suivants: il comporte, dit-il, "une confusion initiale pleine de promesses, dans laquelle se débat un homme courageux, lucide, peu scrupuleux sur les moyens à prendre, et dont l'action résolue laisse espérer les plus belles, les plus complètes solutions; mais les solutions elles-mêmes, vers lesquelles il court en haletant, comme vers une terre promise, seront inévitablement décevantes. II n'y a pas de solutions; seulement des règlements de comptes, et partiels, toujours partiels. Seule l'attente est riche. Seul, le combat. » Quels rapports entrevois-tu entre le roman policier, tel que défini, et un récit comme Un voyage? N'y a-t-il pas, dans les réflexions de Marcel Fournier, quelque chose comme un art poétique, généralisable à bon nombre d'œuvres narratives? ou peut-être applicable particulièrement à tes romans à toi, où l'assurance du personnage cède vite la place à un combat obscur, sans possibilité de «solution » belle ni complète?

G.M. Tu accordes à mes personnages plus d'assurance que je ne leur en reconnais moi-même. Encore une fois, il est possible et même vraisemblable que ce soit toi qui aies raison parce que tu es le lecteur 
et que, moi, je n'ai fait que me débrouiller du mieux que j'ai pu avec tout cela... Bien sûr, le schéma global, en supprimant un certain nombre de détails, pourrait s'appliquer à mes romans et à d'autres romans. Seulement je pense aussi qu'il y a une différence extrêmement importante entre le roman policier et le roman sans qualificatif. Dans le roman policier, les personnages ne sont pas touchés profondément, ne sont pas transformés par les événements. Ils demeurent intacts d'un bout à l'autre. Ce sont des gens qui n'ont pas de problèmes intérieurs; qui ont simplement une sorte de problème en quelque sorte technique à résoudre. Un problème qui devient vital dans la mesure où ils risquent d'y perdre leur vie, mais enfin, cela se passe de façon assez extérieure. Dans l'autre roman, ce sont les personnages qui sont, eux-mêmes, l'aventure. Je les vois comme essentiellement troublés au départ, et tout ce que peut donner le roman, c'est non pas de les délivrer de ce trouble mais de faire que, en cours de récit, ce trouble soit vraiment manifesté.

\section{A.B. Et surmonté?}

G.M. Surmonté, cela, je ne le sais pas. J'ai bien peur, moi, de ces conclusions romanesques qui impliquent une sorte de victoire. Je pense que toute fin de roman, et là je ne parle pas seulement de mes petits romans mais des romans que je lis - et j'en lis beaucoup, j'aime ça! - est nécessairement ambiguë. Car on se trouve devant un personnage qui, dans la presque totalité des cas (et là, je pense à Balzac, à Dostoïevsky, comme je pense à Mauriac, à Malraux, à la presque totalité des romanciers), se découvre nu, à la fin. II est en instance de... Mais il faut ajouter des points de suspension à ce de, autrement il y a malhonnêteté de la part du romancier. Un personnage victorieux est, à mon sens, un faux personnage, un personnage raté.

A.B. Dans ton portrait - magistral - de Marcel Fournier, on trouve les traits suivants: “ll a l'air, oui, disons-le, un peu empêché, comme s'il incarnait divers personnages qui s'interdiraient les uns les autres d'atteindre à l'achèvement. "Puis: "Au total, l'image est assez forte, mais insuffisamment déterminée; ou, comme dirait un linguiste, surdéterminée, offrant à la fois plusieurs possibilités d'interp rétation. » Est-ce que cette définition du personnage ne pourrait pas être appliquée à tes autres héros, Parenteau et Savoie? Et cette surdétermination du personnage n'entraîne-t-elle pas une surdétermination de l'intrigue - les événements et actions extérieurs important moins que les intérieurs, beaucoup plus complexes et difficiles à circonscrire?

G.M. Évidemment, je me suis donné une chance en passant de l'indétermination à la surdétermination. II se trouve que j'ai beaucoup de difficultés à voir mes personnages, et dans le chapitre que tu cites, où je parle de Marcel Fournier, je fais une tentative pour circonscrire un personnage, essayer de lui donner des traits, une démarche, un caractère qui m'échappaient auparavant. D'ailleurs Un voyage, c'est 
peut-être plus une tentative de récit qu'un récit au sens habituel. II y $a$, je pense, d'un côté une indétermination véritable, qui se situe pour moi dans la difficulté de voir. Je ne suis pas un visuel. Cette indétermination peut-elle être considérée aussi comme une surdétermination, comme j'essaie de le dire? Je l'espère, mais je t'avoue que je ne serais pas prêt à me prononcer là-d'essus. C'est une chose que j'abandonne au lecteur. Je ne suis pas prêt à être le lecteur de mes romans. J'ai de la difficulté à me placer devant ces écrtis comme un analyste. Je vois des choses, bien sûr, et j'entends ce que tu me dis, qui me paraît intéressant... S'agit-il d'une surdétermination par insistance sur les événements intérieurs, qui sont plus difficiles à circonscrire, ou d'une indétermination qui serait un problème de la narration?

A.B. Une difficulté à imaginer une action extérieure?

G.M. Oui, cela existe chez moi. J'ai toujours rêvé, je le disais, d'écrire un roman policier, et c'est ce qui m'est absolument impossible.

\section{C - La critique}

A.B. Venons-en à la critique, et aux nombreuses formes de critique que tu as pratiquées, depuis la chronique hebdomadaire jusqu'à l'étude approfondie d'une question ou d'un auteur. Y a-t-il pour toi un hiatus entre la critique de journal et la critique universitaire? As-tu une préférence pour l'une des deux?

G.M. II y a certainement une différence entre la critique de journal et la critique universitaire, comme il y a une différence entre la critique de journal et la critique de magazine, que je pratique depuis quelque temps à Actualité. On parle de la critique au singulier: il y en a toutes sortes de formes. Comme je suis à l'université depuis une quinzaine d'années, j'ai fait des choses que, sans doute, je n'aurais pas faites autrement. Je suis très content que l'université m'ait forcé, en quelque sorte, à faire ce genre de choses; mais quand je fais de la critique universitaire (suis-je sûr d'en faire, au sens fort, c'est une autre question), je ne cesse pas tout à fait d'être journaliste. Ce qu'il y a de nouveau, c'est que je fais des études plus détaillées, qui sont marquées dans une certaine mesure par les interrogations théoriques qui ont cours à l'université, mais j'essaie toujours d'inscrire cela dans une interrogation plus générale, qui se trouve englober ce que je fais dans les différents genres de critique.

A.B. Tu viens de parler des questions théoriques qu'on agite à l'université. Quelle importance accordes-tu à la théorie, en critique littéraire?

G.M. Ah! la théorie, c'est magnifique! J'admire beaucoup ceux qui peuvent faire des travaux qui s'inspirent des théories les plus modernes, 
etc. Seulement il faut bien dire que je suis arrivé tard à ce genre de choses. J'ai fait très peu d'études universitaires, et je ne suis donc pas très à l'aise dans les systèmes théoriques. Je lis, bien sûr, des ouvrages sur la littérature, dont tous comportent une assez large part, de théorie, et cela m'est extrêmement utile, mais peut-être plus à titre de suggestion que de système à appliquer aux œuvres. C'est peut-être pour cela que les grandes œuvres de critique, pour mói, sont des cuvres qui ne font pas la part nette à la théorie, mais qui appliquent une théorie incomplète - qui accepte de ne pas donner tous ses tenants et aboutissants - qui appliquent cette théorie de façon riche, forte et subtile à des œuvres. Je pense en particulier à des gens comme Auerbach ou, plus récemment, Bakhtine, qui ont eu sur moi une très grande influence et que ne sont pas, à mon avis, avant tout des théoriciens même si Bakhtine, dans son dernier livre traduit, parle beaucoup de théorie - d'une façon d'ailleurs qui m'est un petit peu indifférente.

A.B. Tu connais la distinction que fait Starobinski, au début de la Relation critique, entre deux théories: une théorie a priori qui consiste à construire des modèles pour les appliquer ensuite aux œuvres, et une théorie a posteriori qui est une réflexion, une méditation, abstraite et générale, qui découle de la pratique critique, et qui pour lui est tout aussi valable que la précédente. Tu te rallierais plutôt à cette dernière conception de la théorie?

G.M. Oui, et je suis bien content que monsieur Starobinski ait éclarci les choses de cette façon. En effet, c'est à la deuxième définition que je me rallie, et je trouve justement chez Starobinski, dans ce livre qui s'intitule La Relation critique, quelques textes, en particulier son texte sur Rousseau, qui comptent pour moi parmi les plus beaux exemples de critique qui puissent exister. C'est de la critique universitaire, et en même temps ce n'en est pas. Ou plutôt si, c'en est...

A.B. Dans le meilleur sens du terme? (Rires.)

G.M. En fait, je ne refuse pas l'autre du tout. II y a des études très étroitement techniques, certaines études de sémiotique par exemple, qui me paraissent extrêmement valables. Là où je me méfie de la théorie contemporaine, c'est quand je vois un certain nombre de critiques utiliser des théories, qui viennent généralement de France, en leur donnant une portée que la théorie originelle ne justifie pas. De choses très limitées, très strictement définies, ils font presque des systèmes de pensée. Je trouve que, là, il y a souvent abus. Sans oublier, bien sûr, un abus de vocabulaire fréquent, dans l'ensemble de la critique actuelle.

A.B. Quel est, pour toi, l'idéal en critique littéraire? Quelles sont les qualités d'une bonne étude ou d'un bon article? 
G.M. Cela peut être extrêmement divers. Je viens de l'indiquer, j'ai déjà lu des études très étroitement techniques qui me paraissaient admirables. Je pense à une étude, un peu ancienne, faite du point de vue de la grammaire, sur Mallarmé, qui me paraît indispensable. Il y a cela, qui n'est peut-être pas de la critique d'ailleurs, mais comment séparer la critique des diverses opérations qui l'entourent, ou qui parfois y pénètrent? Je pense que, en critique, on peut faire des choses extrêmement différentes les unes des autres.

A.B. Mais toi, quand tu veux rendre compte d'une œuvre quels objectifs te donnes-tu?

G.M. Quand je fais de la critique, il est évident que je ne suis pas dans l'idéal! Ce que j'essaie de faire, c'est de rendre compte du fonctionnement d'une cuvre; j'essaie de le faire en utilisant les moyens du bord, c'est-à-dire les moyens que me donne l'université, que me donne aussi une culture plus générale, et - c'est une pente de ma nature, mais cela me vient aussi de mon expérience de la critique - j'essaie toujours de mettre l'cuvre en rapport avec un ensemble de pensées ou d'actions plus vaste. Et j'essaie de parler «pour le monde»...

A.B. Oui. Cela me rappelle le banal, que tu valorises comme romancier. En critique, ne fondes-tu pas, de façon un peu semblable, ta compréhension de l'œuvre sur le bon sens c'est-à-dire sur des évidences raisonnables et pouvant être immédiatement pariagées - bref, sur la vertu la plus humble mais aussi, la plus efficace, lorsque secondée par l'intelligence? N'y a-t-il pas, chez le romancier comme chez le critique que tu es, un même parti pris de simplicité et une même recherche du vivant dans ses manifestations les plus concrètes, les plus immédiates?

G.M. C'est inquiétant, ça! Je te raconte une anecdote. Un étudiant, maintenant professeur, faisait une étude très savante, très technique en littérature québécoise, et il m'avait dit un jour: "Écoute, j'aimerais bien que tu regardes cela et que tu m'en fasses une lecture de bon sens." J'ai beaucoup ri. Mais il y avait sans doute la quelque chose de réel. Je proposerais - parce que je suis un peu gêné, malgré tout, par l'expression de abon sens" - de la remplacer par "sens commun", en lui donnant une signification un peu plus radicale qu'elle n'a habituellement. II y a, en critique, des choses techniques qui exigent une attention considérable, mais le discours sur la littérature - et là, je parle pour moi - doit atteindre un niveau de communicabilité assez large. Ce qui ne veut pas dire que, en faisant ce genre de textes, on soit assuré d'être compris de tous. J'ai essayé, par exemple, dans mon dernier livre de critique, Le Roman à l'imparfait, d'écrire simplement, et je me suis aperçu en lisant les critiques il y en a eu un certain nombre - qu'on faisait un nombre considérable de contresens à propos de ce livre. Dans certains cas, on m'a fait dire carrément le contraire de ce que je croyais avoit dit très 
clairement. Donc cette ambition de rejoindre le sens commun, dans la réalité, est souvent contrariée. Il y a peut-être là aussi l'effet d'une déformation qui se répand de plus en plus, dans la mesure où les études universitaires se répandent elles-mêmes: le lecteur averti risque de se trouver en quelque sorte déconcerté devant un texte qui veut parler clairement.

A.B. N'est-ce pas parce que la problématique que tu développes, même si elle est formulée très clairement, comporte des présupposés qui ne sont pas présents, au départ, à l'esprit du lecteur?

G.M. Cela est vraisemblable, à tout le moins. Et justement, à la suite de conversations que j'ai eues à propos du Roman à l'imparfait, je me suis dit qu'il serait utile et peut-être même nécessaire de m'arrêter, un de ces jours, sur certains des présupposés qui agissent dans cet essai, pour donner au lecteur un certain nombre d'explications, de développements sur des points qui m'apparaissent, à moi, évidents. Je ne sais pas si j'arriverai à le faire. II y a une certaine impatience, dans ma nature, qui fait que, sur ces présupposés, je veux passer vite; je trouve que ce n'est pas là l'intéressant, pour moi c'est donné. Tu sais, la même chose arrive à des auteurs beaucoup plus considérables que moi. Je pense aux contresens absolument invraisemblables qu'on a faits sur la pensée de McLuhan, sur cette simple formule: le médium, c'est le message, ou encore sur les considérations qu'il fait à propos de l'écriture. Et ces contresens ont cours encore aujourd'hui. McLuhan essaie de rétablir les choses - il n'essaie pas trop, à vrai dire! Mais enfin, ces textes sont quand même clairs, et pourtant les gens s'y perdent. Cela peut venir d'un mot, d'une fausse impression qu'on a au départ et qui se répand comme une traînée de poudre, activée par des préjugés courants.

A.B. Une pensée qui s'exprime dans un langage technique aurait-elle plus de chances d'échapper à cette multiplicité des interprétations? Un langage simple, accessible, est peut-être plus ambigu.

G.M. Je pense que la difficulté est différente. Prenons certains textes que j'ai lus au cours des dernières années et qui partaient d'une pensée technique élaborée. II s'est produit assez souvent, chez certains utilisateurs, un passage du technique au philosophique, qui a mené à des résultats assez aberrants; et parfois même l'utilisation de tels systèmes a constitué, pour des critiques, de véritables interdictions de penser. On avait un système, donc on croyait détenir une vérité, même si l'auteur du système ne l'avait pas proposé comme vérité, et on se laissait aller à répéter, sous une forme apparemment savante, les préjugés les plus solidement enracinés.

A.B. Je voudrais maintenant te poser quelques questions plus précises sur tes ouvrages. D'abord sur le premier que tu as publié, Une littérature qui se fait. II y est question, à propos de Saint-Denys Garneau, de la 
vibration vitale, notion empruntée à Claudel. Tu écris: “Cette dernière, va-et-vient d'une surabondance de vie, constitue le rythme lui-même de la création, dans un équilibre libérateur des contraires; tandis que la vibration à laquelle est livré Saint-Denys Garneau est fascination successive par deux inconciliables, et se résout finalement dans le perpétuel recommencement du cercle. " Faut-il entendre par là que la création, chez Saint-Denys Garneau, est empêchée, entravée par la discordance ontologique? N'y a-t-il pas le danger de conclure de l'échec humain à l'échec esthétique et de sombrer ainsi dans une sorte de moralisme? D'autre part, ton analyse est-elle tout à fait convergente avec celle de Jean LeMoyne qui parle de dualisme à propos de l'œuvre - et de la vie - de Saint-Denys Garneau? Le dualisme, estce bien cette fascination successive par deux inconciliables?

G.M. Le dualisme dont parle Jean LeMoyne n'est pas tout à fait cette fascination dont je parle, mais les deux observations, celle de LeMoyne et la mienne, vont dans le même sens. II faut situer ces observations dans l'époque. Chez ceux qui étaient le plus près de Saint-Denys Garneau, on avait certaines réserves quant à la réussite totale de l'œuvre. La réserve chez LeMoyne, et chez moi aussi, s'exprimait dans la perspective d'une recherche de l'unité. Unité qu'exprime aussi la vibration claudélienne. Une œuvre qui, thématiquement à tout le moins, ne semblait pas pouvoir atteindre à cette unité semblait donc souffrir d'une sorte de défaut ontologique qui affectait à la fois l'homme et l'œuvre. Je ne renie pas complètement cette analyse, mais ma position a tout de même changé considérablement dans la mesure où je crois, aujourd'hui, que les inconciliables peuvent autant constituer un principe de construction de l'œuvre, que signaler un déficit. Le Saint-Denys Garneau que je lis aujourd'hui, et qui devient pour moi, plus encore qu'à l'époque, un très grand écrivain, c'est celui, justement, des inconciliables, celui qui n'atteint pas à l'unité mais qui travaille sur un autre plan, beaucoup plus moderne, un plan de dirais kafkaïen, qui implique non pas la vibration, ou la résolution des contraires, mais un jeu dans ce que j'appellerais les interstices du langage, procédant par glissements, déplacements subtils, imposant une façon de faire, d'aller, qui n'a plus rien à voir avec le dualisme traditionnel. Ouf !... Comme tu vois, mon Saint-Denys Garneau est devenu très différent de celui que je lisais à l'époque.

A.B. Ce Saint-Denys Garneau déchiré entre les incompatibles, y a-t-il moyen de le mettre en rapport avec le paradoxe baroque que tu évoques à propos d'autres écrivains de sa génération, notamment de Rina Lasnier?

G.M. II y a quand même une différence. Le baroque chez Rina Lasnier se propose dans des formes en quelque sorte évidentes.

A.B. II est thématisé? 
G.M. Oui, très fortement, et il s'interdit ce que j'appelais tout à l'heure le glissement chez Saint-Denys Garneau, glissement que j'observe surtout dans un texte qui est un chef-d'œuvre et qui a d'ailleurs été analysé admirablement par Albert Béguin : le Mauvais Pauvre. C'est, pour moi, le chef-d'œuvre de Saint-Denys Garneau. Encore une fois, je le lisais à l'époque sur un plan plus psychologique. Maintenant je le lis dans ses opérations langagières - qui ne sont pas sans rapport avec celles de la vie. Dans la plupart des analyses qu'on a données jusqu'à maintenant de Saint-Denys Garneau, il me semble qu'on a fait trop souvent l'économie de ce qui se passe dans le langage, et qui est extrêmement fort, saisissant, neuf.

A.B. Dans le Temps des poètes, tu fais de Saint-Denys Garneau, Alain Grandbois, Rina Lasnier et Anne Hébert les initiateurs de la modernité littéraire au Québec. Quelle est ta définition de la modernité, et quelle place y assignes-tu au courant formaliste?

G.M. Enfin une question simple! (Rires.) La modernité, bien sûr, c'est une tarte à la crème, et la première chose à faire est de mettre le mot au pluriel. C'est une tendance que j'ai souvent, mais ici c'est particulièrement important. La définition que je peux donner de la modernité, je la tire, sauf erreur, de Michel Foucault, en simplifiant peut-être démesurément les choses. II s'agit, au premier chef, de la modernité du dix-neuvième siècle.

Modernité dont nous vivons encore : là-dessus, il ne faut pas se faire d'illusions. Il y a deux éléments à mon avis et, je pense, à celui de Foucault, qui définissent cela. C'est d'abord l'historicité, le sens du mouvement temporel, qui n'existait pas dans la pensée du dix-huitième ou du six-septième siècles. L'autre trait qui caractérise cette modernité serait le double fond, c'est-à-dire la conviction que sous le sens premier se cachent toujours d'autres sens. Cela rejoint d'ailleurs ton idée de la vie intérieure, dans une certaine mesure. Voilà donc, pour moi, les deux traits essentiels de la modernité, qui est la modernité du dix-neuvième siècle et qui a commencé à apparaître, ici, uniquement avec la Relève. Cela, c'est extrêmement important. Mais il y a l'autre modernité, la modernité très moderne, que tu définis par le formalisme. Elle implique autre chose qui est la disparition de la voix, pour emprunter les termes de Derrida; l'écriture supplante la voix, la parole. Elle supprime d'ailleurs un tas de choses en passant: l'auteur... enfin, le “bag»! Bon. Je pense que, dans une certaine mesure, Saint-Denys Garneau participe à cette deuxième modernité, par ses textes de prose en particulier. Et c'est cela qu'on commence à percevoir aujourd'hui.

A.B. Puisque tu parles de voix, je remarque que, dans plusieurs textes critiques, tu accordes beaucoup d'importance à la chose. Au début de la recension des Indes, de Marguerite Duras, tu écris : "On en revient toujours là : un écrivain a une voix, la sienne, ou n'en a pas; et nom- 
breux sont actuellement les romanciers qui font grand tapage avec la voix des autres. "Toujours dans les Bonnes Rencontres, tu écris que “les murs (de l'institution littéraire) sont lézardés; restent les voix "après quoi tu cites Jean Grosjean. La voix dont tu parles est-elle bien, comme l'affirme Derrida, le contraire de l'écriture? Suppose-t-elle une métaphysique de la présence, un humanisme?

G.M. Cette question me rend perplexe. Cette métaphysique de la présence est-elle pour moi capitale? Je suís tente de dire oui quand je pense au mot présence, et non quand je pense au mot métaphysique. Le texte de Grosjean que j'ai cité exprime une tentation. II y a toujours quelque chose en moi qui va aller, au niveau de l'émotion ou du sentiment plutôt qu'à celui de la pensée, vers cette conception de la voix qui touche, par certains côtés, la métaphysique. Mais d'un autre côté je pense que, dans le texte de moi que tu cites, la voix est autre chose, peut-être de plus général, et qui peut se faire entendre dans le règne de l'écriture autant que dans celui de la parole. Tu as cité Derrida : je pense que Derrida est justement un écrivain qui a une voix très reconnaissable, extrêmement personnelle. Peut-être qu'au fond le mot voix, tel que je l'employais, voulait dire: style. Mais je déteste le mot style, parce que ses connotations sont, à mon avis, étroitement techniques alors que le mot voix, pour moi, exige beaucoup plus.

A.B. La voix suppose une présence, mais il y a tout de même une part de négation dans l'activité même de l'écriture puisque tu parles, toujours à propos de Saint-Denys Garneau, d'une "souveraine interdiction qui habite les profondeurs du langage et à laquelle se heurte le poète", comme s'y étaient heurtés avant lui Nerval, Baudelaire, Mallarmé et Breton (Le Temps des poètes). Pourrais-tu préciser la nature de cette interdiction, et dire si elle est liée à la modernité, ou consubstantielle à toute poésie?

G.M. Je ne crois pas qu'elle soit consubstantielle à toute poésie. II me semble que cette interdiction est liée, justement, à la modernité telle que j'essayais de la définir tout à l'heure, la modernité du dix-neuvième siècle, dans la mesure où s'y développe un conflit extrêmement grave, extrêmement profond entre un désir de transparence qui serait le désir de la voix au sens fort, au sens derridien de la parole, et le sentiment que cette transparence est devenue impossible, qu'on est entré dans ce que Foucault appellerait une autre épistémè, qui interdit de croire que cette transparence soit possible.

A.B. Le Temps des poètes était consacré exclusivement à la poésie, comme son titre l'indique, et les Bonnes Rencontres le sont à la prose. Dans la présentation de cet ouvrage, tu affirmes que la poésie cinvite à des manières d'écrire très différentes de celles que suggère la prose. En quoi consiste cette différence? $Y$ a-t-il des précautions particulières à prendre, face à la poésie? La prose autorise-t-elle des analyses plus poussées? 
G.M. C'est d'abord une question de quantité. Quand tu parles de poésie, tu dois ajouter au texte parce que, par définition, la poésie, et surtout la poésie moderne, est peu nombreuse. Tandis que, quand tu parles de la prose, tu dois réduire le texte. Dans la mesure où il y a, dans toute forme de critique, une certaine concurrence entre le texte dont tu parles et le texte que tu fabriques, cette différence quantitative est importante. Parler de poésie implique une sorte d'invention verbale que n'exige pas le travail qu'on peut faire sur la prose. II faut dire aussi que la prose, au contraire de la poésie, nous permet de parler de tout. La poésie moderne, celle du vingtième siècle, est une poésie plutôt laconique et même, dans une certaine mesure, hermétique, tout le monde sait cela; et on peut essayer de déplier ce texte, on peut même essayer, comme on le faisait dans une étude que j'ai lue récemment, de trouver une signification sociale à des poèmes comme ceux de Paul-Marie Lapointe. Mais c'est toujours assez difficile. Tandis que la prose, par définition, c'est une sorte de lieu commun qui nous permet de faire toutes sortes de rapports entre ce que j'appellerais la prose de l'écrit et la prose du monde, c'est-à-dire le langage proliférant au milieu duquel nous vivons.

A.B. J'aurais une dernière question à te poser sur le Temps des poètes, et elle nous permettra de revenir brièvement à tes romans. A propos des automatistes, tu affirmes que «le malheur des révoltes au Canada français, poétiques ou autres, est de s'attaquer à un ordre culturel qui se défend mal ou ne se défend pas, ou qui plus justement n'a pas de quoi riposter". Dans les Bonnes Rencontres par ailleurs, louant l' "authentique révolte humaine" de Mounier, tu lui opposes nos pratiques locales: "Nos révoltes se font mal; parfois à l'encontre de la foi, et presque toujours, même quand la foi demeure, dans un désordre affectif et intellectuel qui fausse pour longtemps les rapports de l'homme et du chrétien, s Comment définirais-tu la bonne révolte? Cette révolte est-elle radicalement impossible chez nous et, si oui, la révolte maladroite qui en tient lieu est-elle à rejeter? D'autre part, la révolte de Claude Savoie (Le Poids de Dieu) te semble-t-elle souffrir de la situation que tu dénonces, c'est-à-dire de l'absence de riposte de l'ordre culturel auquel elle s'attaque?

G.M. La bonne révolte... D'abord, j'encaisse. (Rires.) Ensuite, j'essaie de répondre. - Le problème des révoltes, dans notre milieu, vient de ce que ce milieu est le moins traditionnel qui soit, je pense, dans le monde actuellement. On a souvent parlé du Québec un peu ancien, du Québec d'avant la Révolution tranquille comme d'un milieu traditionnel. Je trouve que c'est un contresens. La tradition ici a toujours été extrêmement fragile. C'est un milieu où on n'hérite pas, où on respecte très peu les aînés, où on ne cesse pas de secouer le cocotier. Et cela n'est pas nouveau; il en a toujours été un peu ainsi. 


\section{A.B. Même sous Duplessis?}

G.M. Même sous Duplessis, bien sûr. Je pense qu'on se fait, de la situaation sous Duplessis, une image un peu simple. Il suffit de lire ce qui s'écrivait à l'époque, dans divers journaux, dans les revues. C'était aussi violent que ce qui s'écrit aujourd'hui; dans un autre style, selon d'autres coordonnées. De toute façon, la tradition ici m'a toujours paru extrêmement faible, et d'ailleurs, on a vu ce qui s'est passé depuis '60. Tout est disparu comme par enchantement. II n'y a pas eu de lutte, de chose très grave, d'affrontement. On a dit : bon, la religion, ce n'est peut-être pas ce qu'il nous faudrait - la religion catholique -; eh bien, on a soufflé sur le château de cartes, et l'institution religieuse qui nous paraissait la plus puissante au monde s'est recroquevillée dans son coin. On n'a pas eu de grand débat là-dessus.

A.B. Comment expliquer alors la révolte très profonde des écrivains de la Relève contre le catholicisme conservateur et contre la société de l'époque.

G.M. Je pense que c'était une révolte contre le rien, contre le vide, et non contre des choses très réelles et puissantes. Voilà pourquoi il est difficile, à mon avis, de faire ce que tu appelles ironiquement la «bonne révolte". Pour faire une révolte, il faut avoir une tradition qui soit, dans une certaine mesure, respectée. Quand cette tradition se déclare faible ou vide, la révolte ne trouve plus d'aliment.

A.B. C'est la raison pour laquelle il n'y a pas d'affrontement réel entre l'abbé Savoie et le curé Marquis?

G.M. Absolument pas, en effet. Comme il ne peut y avoir d'affrontement très réel dans mes autres romans non plus.

A.B. Il y a même une sorte de complicité entre l'abbé Savoie et le curé Marquis.

G.M. Je pense que le curé Marquis est d'ailleurs le père que Claude Savoie n'a pas eu, et que cette reconnaissance de l'un par l'autre, surtout de l'abbé Savoie par le curé Marquis, est une des choses qui, peut-être, permettent à l'abbé Savoie de survivre.

A.B. Passons à une autre question, sur le Roman à l'imparfait, cette fois. A la fin de l'ouvrage, dans un bref parallèle entre le Canadien-anglais Mordecai Richler et Gérard Bessette, tu écris que le premier "appartient à une culture où l'ordre historique conserve ses pouvoirs; Gérard Bessette, non ", ce qui expliquerait l'impossibilité pour ce dernier d'écrire un roman "où le temps gouverne les actions et mûrit les personnages". Qu'est-ce qui prive notre culture de cet "ordre historique" et nous condamne, en quelque sorte, au "roman à l'imparfait"? Et entrevois-tu une évolution possible de la situation? Un roman comme La Grosse Femme d'à côté est enceinte ne restaure-t- 
il pas la fonction représentative sur laquelle est fondée la tradition du grand roman réaliste?

G.M. Quand je parle de l'ordre historique, il s'agit de l'ordre historique tel qu'il a été fondé par le dix-neuvième siècle. C'est quelque chose d'essentiellement moderne et je rappellerai, à ce sujet, la remarque de McLuhan : le Canada français est passé à pieds joints par-dessus le dix-neuvième siècle. Nous ne sommes pas entrés dans cet ordre historique.

A.B. Nous avons sauté par-dessus l'industrialisation, la société industrielle?

G.M. Oui. L'ordre historique, c'est un ensemble. C'est le triomphe d'une certaine forme, d'une certaine vision du monde qui se manifeste autant dans l'ordre industriel que dans les façons d'écrire. Donc, nous n'avons pas eu de dix-neuvième siècle, sinon de façon parcellaire, et très en retard. Notre dix-neuvième siècle romanesque, il a duré dix ans : c'est Gabrielle Roy, Germaine Guèvremont, Roger Lemelin et Ringuet. Ensuite, on arrive à tout à fait autre chose. Mais il est intéressant de voir ce qui se passe dans les travaux historiques eux-mêmes. Nous allons trouver là une illustration̈s encore plus saisissante de ce que j'essaie de dire dans le Roman à l'imparfait. Il y a eu le chanoine Groulx, qui est un historien à la manière de Bossuet, en somme, c'est-à-dire du grand dessein de Dieu sur les sociétés: une histoire qui refuse les valeurs modernes de l'historicité. Voilà donc notre dix-septième siècle, que nous avons au vingtième: c'est curieux! Après cela, nous sommes passés très rapidement à l'historiographie, disons post-moderne, qui, elle, est volontairement parcellaire, qui ne va plus dans les ensembles comme on y allait au dixneuvième siècle. Un bon exemple de cette façon de faire l'histoire, c'est le premier tome de l'ouvrage de Durocher et autres, sur l'histoire du Québec, où la perspective unitaire est abolie. Le découpage de l'ouvrage l'indique nettement. Nous sommes donc passés, en quelques décennies, de l'histoire très ancienne à l'histoire très moderne.

A.B. II y a tout de même eu ce qu'on a appelé l'école d'histoire de Montréal: Maurice Séguin, Michel Brunet, Guy Frégault.

G.M. Oui : qui correspond assez exactement aux dix années du roman. Mais le chanoine Groulx, tout de même, ce n'est pas loin... D'autre part, la Grosse Femme d'à côté est enceinte constitue, je pense, un excellent argument pour ma thèse! Tu as raison d'y voir des ingrédients réalistes, mais ce qui m'apparaît aussi extrêmement significatif, c'est qu'on n'y sent pas le passage du temps, de l'histoire. Cela se situe à une certaine époque; je pense que cela pourrait se situer maintenant et il y aurait très peu de différence. Et surtout, il y a cette histoire des tricoteuses, qui enveloppe le récit dans une atmosphère et dans un processus mythiques. Si on compare la Grosse Femme d'à 
côté est enceinte à un roman de Balzac, on constate tout de suite une énorme différence.

A.B. II s'agit, en fait, d'une chronique qui nous présente une tranche de vie très limitée dans le temps, et on ne peut donc pas voir mûrir les personnages.

G.M. J'ajouterais qu'on se trouve dans l'invraisemblable. Et le défaut de ce roman, qui est par ailleurs remarquable, c'est de n'avoir pas consenti jusqu'au bout à l'invraisemblable. Dans ce roman, Tremblay prend le mythe de la fécondité, qui est un mythe canadien-français par excellence, et il le renverse: la grosse femme d'à côté ne veut pas avoir des enfants par devoir, devoir qui est inclus dans le mythe traditionnel de la fécondité ; elle veut avoir un enfant par plaisir, pour réussir en quelque sorte une œuvre esthétique, pour la beauté du monde. Si Tremblay avait voulu faire de ce désir une espèce d'explosion, on aurait eu quelque chose d'absolument extraordinaire, dans le genre de ce que peuvent proposer les Sud-Américains actuellement. II s'est arrêté trop tôt.

A.B. Maintenant, une question plus générale sur ton attitude devant la littérature québécoise. Depuis à peu près trente ans, tu as suivi attentivement cette «littérature qui se fait», la nôtre, et tu as assisté à d'assez étonnantes métamorphoses. L'intérêt et la confiance que tu manifestais à l'origine, et qui s'exprime par exemple dans l'Avertissement d'Une littérature qui se fait, sont-ils restés intacts au cours des années? Vois-tu notre avenir littéraire de la même façon que tu le voyais au début des années ' 50 ?

G.M. Trente ans, c'est un bail! La confiance, oui, elle est intacte, dans la mesure où elle était quand même mesurée à l'époque, si je me réfère à l'Avertissement d'Une littérature qui se fait. Mais elle se formulerait de façon sans doute différente. La façon dont j'imaginais les œuvres à venir, en particulier à la fin de ma petite histoire du roman, serait transformée aujourd'hui. Quand j'écrivais ces textes, je les écrivais selon une certaine conception de l'œuvre et de l'histoire qui faisait que nous allions vers l'unité, vers ce que tu as appelé à plusieurs reprises des solutions. Aujourd'hui, je vois l'avenir sous la forme d'un éclatement continué et d'une diversité.

A.B. Tu as donc passé toi aussi, comme le roman québécois, du dixneuvième au vingtième siècle. Ce changement, dans la façon de te représenter notre avenir littéraire, et sans doute la littérature ellemême, a-t-il constitué pour toi un déchirement?

G.M. Non, pas du tout. Je peux avouer, en toute franchise, une certaine nostalgie des formes anciennes du roman; j'y reviens souvent, et je pense d'ailleurs qu'elles ne sont pas mortes. Elles sont pour nous presque hors d'atteinte, mais je ne vis pas seulement au Québec, je vis aussi dans le monde occidental, et il se produit encore dans ce 
monde des choses qui sont dans une relative continuité avec le roman dit traditionnel, des cuvres où du moins la rupture se fait de façon différente. Non, je ne regrette rien : les regrets ne sont pas dans ma nature. Et je n'ai pas non plus de nostalgie dans la mesure où cette littérature demeure aussi mon présent. Je pense qu'on peut vivre plusieurs présents, et c'est peut-être cela que nous sommes appelés à faire aujourd'hui. C'est un petit peu embêtant, parce que, encore une fois, ce que nous suggéraient les études de notre jeunesse était très différent, c'était une perspective d'unité. Aujourd'hui nous sommes appelés à vivre à plusieurs époques en même temps. Moi, je trouve cela plutôt amusant. Je puis lire côte à côte Balzac, qui m'intéresse beaucoup ces temps-ci, et Réjean Ducharme, sans éprouver aucune gêne.

A.B. La confiance - mesurée - que tu as en notre avenir littéraire, tu l'as aussi dans le destin même de notre collectivité?

G.M. Oui. C'est-à-dire que nous sommes dans les choses temporelles, n'est-ce pas? Donc il ne faudrait pas essayer de figer l'espoir dans des réalisations anticipées. Ce serait extrêmement dangereux, à la fois pour notre littérature et pour notre collectivité. Nous sommes en processus, nous sommes en aventure, et l'espoir que je peux avoir et pour la littérature et pour la collectivité, il est fondé sur ce que nous sommes et sur ce que nous avons maintenant. Comme mon destin est lié dans une grande mesure à celui de cette collectivité, ce serait commettre un suicide de croire que cette histoire va s'arrêter. D'ailleurs cela me paraîtrait tout à fait invraisemblable. 